\title{
THE ANNUAL MEETING IN NEW YORK
}

The thirty-fifth Annual Meeting of the American Mathematical Society was held in New York City, from Wednesday to Monday, December 26-31, 1928, in conjunction with the meetings of the American Association for the Advancement of Science and the Mathematical Association of America. All the sessions of the Society were held at Columbia University: the Gibbs Lecture in the Casa Italiana, the Symposium on Quantum Mechanics in Room 401 of the Physics Laboratories, all other sessions in Barnard College. The meeting opened on Wednesday evening with a session of the Board of Trustees at the Hotel Lincoln (the headquarters of the mathematical organizations). Thursday was devoted to sectional sessions of the Society. The third award of the Bôcher Memorial Prize was announced at a general session on Friday forenoon; this session was followed by the annual business meeting, at which trustees, officers and members of the Council were elected. The first part of Friday afternoon was devoted to a joint session of the Society, the Mathematical Association and Section A of the American Association for the Advancement of Science; this session was followed by the sixth Josiah Willard Gibbs Lecture under the joint auspices of the Society and the A.A.A.S. On Saturday morning the Society held a joint session with Section $\mathrm{K}$ of the A.A.A.S. (Social and Economic Sciences); the Mathematical Association also held its sessions on Saturday. Monday was devoted to a Symposium on Quantum Mechanics, held under the joint auspices of the American Mathematical Society and the American Physical Society.

The joint dinner of the mathematical organizations, held on Friday evening in the Men's Faculty Club of Columbia University, was attended by about one hundred ninety persons. Professor Dunham Jackson was toastmaster.

The mathematicians and their guests were invited to the 
various general lectures and receptions held by the A.A.A.S. They enjoyed especially a complimentary concert given to the attending scientists in Carnegie Hall on Sunday afternoon by the New York Philharmonic-Symphony Society.

The attendance at this meeting (the largest on record) included the following two hundred eighty-two members of the Society:

C. R. Adams, R. B. Adams, Adshead, A. A. Albert, E. S. Allen, Joseph Allen, Altshiller-Court, R. L. Anderson, L. B. Andrews, R. C. Archibald, R. G. Archibald, C. L. Arnold, H. E. Arnold, Ashcraft, Atchison, R. W. Babcock, Bacon, Bamforth, Barnett, Beetle, A. A. Bennett, Theodore Bennett, A. C. Berry, William Johnston Berry, Birkhoff, Blumenthal, Bowden, Bower, Bradshaw, Brinkmann, Bristol, Burington, Burkett, Cairns, Caldwell, B. H. Camp, A. D. Campbell, Carlen, R. D. Carmichael, E.T.Carroll, Carruth, A.G.Clark, Clarke, L.W. Cohen, Congdon, Coolidge, Cope, A. H. Copeland, L. P. Copeland, Cramlet, Currier, Dadourian, Darkow, J. E. Davis, Decker, Dehn, Denton, Dimick, DoBell, Dorwart, Dorweiler, Dresden, Edmondson, Eisenhart, W. W. Elliott, Engstrom, H. P. Evans, Farnum, Feinler, Fiske, Fite, D. A. Flanders, Focke, Fort, A. L. Foster, M. C. Foster, Philip Franklin, Frink, Frumveller, Fry, C. A. Garabedian, Gehman, Gergen, J. L. Gibson, Gill, D. C. Gillespie, Gilman, Glasgow, J.W. Glover, P. H. Graham, A. A. Grant, Gronwall, C. C. Grove, Hall, Hallett, Hammatt, Hansell, J. G. Hardy, E. R. Hedrick, Robert Henderson, Hickson, Hille, Himwich, Hofmann, Hollcroft, J. C. Hughes, O. M. Hughes, Huntington, Dunham Jackson, Jeffery, M. I. Johnson, R. A. Johnson, Jonah, E. H. Jones, Kasner, Kazarinoff, O. D. Kellogg, Keyser, Kholodovsky, B. F. Kimball, Rose Klein, Kline, Kramer, Lambert, Landers, Landis, Langman, Larew, Lefschetz, D. H. Lehmer, Lehr, Leib, Leinbach, Lepeshkin, Harry Levy, F. P. Lewis, Linehan, Ling, Littauer, Longley, Lotka, Lubben, Lubin, McGiffert, MacColl, MacCreadie, MacInnes, Marden, Mathewson, S. P. Mead, Mears, Meder, J. E. Merrill, E. J. Miles, Norman Miller, H. H. Mitchell, Mode, Molina, C. N. Moore, R. L. Moore, Moriarty, Frank Morley, C. C. Morris, Richard Morris, D. S. Morse, Marston Morse, Morton, Murnaghan, F. H. Murray, Nassau, C. A. Nelson, Newman, Ogburn, Olson, Oppenheim, Ore, Osgood, F. W. Owens, H. B. Owens, Paradiso, Patterson, Pattillo, Paxton, Pell-Wheeler, F. W. Perkins, T. S. Peterson, Pfeiffer, H. B. Phillips, Pierpont, Pixley, Pooler, Poritsky, Post, R. G. Putnam, Rainich, Rambo, Ramsdell, Raudenbush, Rawles, Reddick, C. J. Rees, M. S. Rees, R. G. D. Richardson, D. E. Richmond, Rider, Risselman, Ritt, Robertson, Robison, E. D. Roe, Roever, Roman, Roos, Rorer, J. E. Rowe, Ruger, Rupp, Saurel, Sauté, Schoonmaker, Seely, Seidlin, Serghiesco, Shaub, Sheffer, Shewhart, Siceloff, Silverman, Simons, Singer, Slaught, Slobin, Slotnick, Smail, A. H. Smith, C. E. Smith, W. M. Smith, Stafford, M. E. Stark, Stetson, Stocker, Stokes, M. H. Stone, Struik, Tamarkin, J. M. Thomas, J. E. Thompson, L. T. E. Thompson, Trjitzinsky, B. M. Turner, Tyler, Vallarta, Vandiver, Van Vleck, 
Waddell, Wahlert, G. W. Walker, C. M. Walsh, J. L. Walsh, H. E. Webb, Wedderburn, D. W. Weeks, Weida, Weisner, M. E. Wells, Weyl, A. M. Whelan, H. S. White, Whited, Widder, Wiener, C. E. Wilder, F. B. Williams, W. L. G. Williams, John Williamson, A. H. Wilson, E. B. Wilson, E. W. Wilson, W. A. Wilson, J. W. Young, Margaret M. Young, Zariski.

At the meeting of the Council, the following fourteen persons were elected to membership in the Society:

Mr. John Geoffrey Adshead, Dalhousie University;

Professor Herbert Eli Arnold, Wesleyan University;

Professor May Margaret Beenken, State Teachers College, Oshkosh;

Professor Ludwig Bieberbach, University of Berlin;

Mr. Leo Branovan, University of Minnesota;

Professor James Edward Donahue, University of Vermont;

Professor Karl Wilmot Folley, Trinity College;

Miss Rose Klein, Washington Irving High School;

Miss Erma M. Leinbach, Sweet Briar College;

Mr. Allen Shelly McMaster, University of Colorado;

Dr. Edward Aaron Saibel, University of Minnesota;

Mr. Howard Elmer Wahlert, Washington Square College, New York University;

Miss Dorothy Walcott Weeks, Wellesley College;

Professor Hermann Weyl, Princeton University.

The following were elected to membership as nominees of Allyn and Bacon:

Mr. Hubert Banks Huntley, Brown University;

Mr. William Clemmer Mitchell, University of Cincinnati;

Mr. George Sauté, Harvard University;

as nominees of the National Life Insurance Company of the United States of America:

Mr. Aubrey Wilfred Landers, Brown University;

Dr. William Maier, University of Chicago;

as nominees of the University of Michigan:

Mr. Ruel V. Churchill, Mr. Shirley E. Field, Mr. Nevin C. Fisk, Mr. Walter O. Menge, Mr. Theodore E. Raiford, all of the University of Michigan;

as nominees of the Westinghouse Electric and Manufacturing Company:

Mr. Charles LeG. Fortescue, Mr. Clinton R. Hanna, Mr. George R. Karelitz, Mr. John F. Peters, Mr. Nicholas Rashevsky, Mr. Morris Stone, Mr. Demetry I. Vinogradoff, all of the Westinghouse Company's Engineering Department at East Pittsburgh.

The ordinary membership in the Society is now 1796, in- 
cluding 178 nominees of sustaining members and 80 life members. There are also 38 sustaining members. The total attendance of members at all meetings during the past year was 769 ; the number of papers read was 439 . The number of members attending at least one meeting was 560 . At the annual election 265 votes were cast.

The reports of the Treasurer and of the auditors (Professors H. W. Reddick and Paul Saurel) showed a balance of $\$ 4709.55$ exclusive of the balances in the Bulletin, Transactions, Colloquium, Journal and special funds, and of the life membership reserve. The Society's Endowment Fund now has securities of par value $\$ 75,000$ yielding an annual income of $\$ 3515$; sustaining membership fees for the year amounted to $\$ 4810$. The amount received from sales of the Society's publications was $\$ 6776.38$. The trustees adopted a budget for 1929 showing estimated expenditures and receipts as $\$ 32,245.42$ and $\$ 30,855.42$ respectively. During 1928 members made special contributions, amounting, approximately, to twelve hundred dollars, which wiped out the deficit on the year's business; it is hoped that these contributions will be increased this year so that the deficit anticipated for 1929 may be covered.

The Librarian reported that the Library of the Society now contains 7773 volumes.

At the annual election, which closed on Friday morning, the following trustees and officers and other members of the Council were chosen:

Board of Trustees, Professors J. L. Coolidge, W. B. Fite, E. R. Hedrick, R. G. D. Richardson, Oswald Veblen.

President, Professor E. R. Hedrick.

Vice-President, Professor Solomon Lefschetz.

Associate Secretary, Professor Arnold Dresden.

Member of the Editorial Committee of the Bulletin, Professor W. R. Longley.

Member of the Editorial Committee of the Transactions, Professor Dunham Jackson.

Members of the Editorial Committee of the Colloquium Publi- 
cations, Professors G. D. Birkhoff, R. L. Moore, J. W. Young. Members of the Council, Professors H. F. Blichfeldt, Daniel Buchanan, B. H. Camp, J. R. Kline, E. P. Lane.

The tellers appointed to count the ballots were Professors C. R. Adams and R. A. Johnson.

At the annual business meeting of the Society on Friday morning Vice-President Kellogg presented the following resolution, which was unanimously adopted: The American Mathematical Society desires to express to the President and Trustees of Columbia University, its thanks for their hospitality in connection with the present meeting, and its appreciation of the effective measures taken to make the meeting successful.

The following resolution in memory of the late Dean Fine, also presented by Vice-President Kellogg, was unanimously adopted by the Society:

The members of the American Mathematical Society have learned with profound sorrow of the death of Dean Henry Burchard Fine on Saturday last, December 22. Dean Fine was a member of this Society from its earliest days; he was its president in 1911 and 1912, and a member of its Council for many years thereafter. His whole life was devoted with rare singleness of purpose to the cause to which this Society is dedicated, the building up of mathematical science in America. He was an inspiring teacher; his influence was undeviatingly exerted for rigorous thinking and for the highest standards of scientific honesty and quality of production. These things he fostered not only by the force of his personality but also by his keen appreciation of talent in others and his selfless furthering of the opportunities and careers of those so endowed. The department of mathematics at Princeton is a monument to the forty-nine years of patient and wise planning which Dean Fine devoted to its building. The Society desires to express its sense of great loss in the death of its honored member, and order that this minute be entered in its records, also that copies be sent, through the President, to Princeton University, and to his daughter, his brother, and to his sisters. 
The following appointments were reported: to represent the Society at the inauguration of President H. N. Davis, of the Stevens Institute of Technology, on November 23, 1928, Vice-President O. D. Kellogg; to represent the Society at the American Mining Congress on December 5-8, 1928, Dr. C. E. Van Orstrand.

At the meeting of the Board of Trustees it was decided to raise the prices of back volumes of the Transactions and the Bulletin and, effective January 1, 1930, to raise the price of current volumes of the Transactions to $\$ 8$.

At the meeting of the Council, Professor James Pierpont was named to succeed President Virgil Snyder as one of the three representatives of the Society in the Division of Physical Sciences of the National Research Council. Professors E. V. Huntington, chairman, Dunham Jackson and H. E. Slaught were appointed as a committee to advise the printers as to forms of mathematical type.

It was voted to thank Professor Tomlinson Fort for his services on the Committee on Sustaining Memberships.

The Council decided to grant the request of the San Francisco Section that the meetings on the Pacific Slope be hereafter designated as regular meetings of the Society.

Professors E. B. Stouffer, chairman, M. H. Ingraham and H. L. Rietz were appointed a Committee on Program for the Western Meetings of the Society.

It was announced that the seventh Josiah Willard Gibbs Lecture would be held at Des Moines in December 1929 in connection with the Western meeting of the Society and the sessions of the American Association for the Advancement of Science and that a committee consisting of Professors O. D. Kellogg, chairman, E. B. Stouffer and Warren Weaver had been appointed to recommend a lecturer to the Council.

It was announced that the title of Professor R. L. Moore's colloquium lectures, to be delivered in Boulder at the Summer Meeting of 1929, will be Point set theory, and that Professor Lefschetz will deliver the colloquium lectures, at 
Brown University at the summer meeting of 1930 , the title to be announced later.

The general session on Friday morning opened with an address by Professor W. F. Osgood on Maxime Bôcher. Immediately afterwards the chairman, Professor E. B. Van Vleck announced that the Council, upon recommendation of a committee consisting of Professors Bell, Carmichael and Lefschetz, had made the third award of the Bôcher Memorial Prize to Professor J. W. Alexander, of Princeton University, for his memoir entitled Combinatorial analysis situs, published in volume 28 of the Transactions of this Society. Professor Alexander was unfortunately prevented by illness from being present to receive the award. The session closed with an invited address by Professor James Pierpont, entitled On the motion of a rigid body in a space of constant curvature.

At the joint session of the Society, the Mathematical Association, and Section A, held on Friday afternoon, the following papers were read:

I. The relation of statistics to modern mathematical research, by Professor Dunham Jackson, retiring Vice-President of Section A. This paper was published in Science for January $18,1929$.

II. The heroic age of geometry, by Professor J. L. Coolidge. This address, delivered upon invitation of the Society and the Mathematical Association, was published in this Bulletin, for January-February, 1929.

The sixth Josiah Willard Gibbs Lecture, entitled $A n$ introduction to the theory of numbers, was to have been delivered by Professor G. H. Hardy, of the University of Oxford. Shortly before the hour announced for this lecture it was learned that illness would make it impossible for Professor Hardy to be present. Professor H. W. Brinkmann, of Harvard University, very graciously consented to present the material of the lecture. The lecture will be printed in full in an early issue of this Bulletin.

The attendance at the joint session and at the Gibbs Lecture ran well over 300 . 
On Monday was held a symposium on quantum mechanics, under the joint auspices of the American Mathematical Society and the American Physical Society. This was in the form of invited addresses, each followed by discussion. The program was as follows:

Physical meaning of wave mechanics, by Professor J. C. Slater. Discussion led by Dr. W. F. G. Swann and Professor E. U. Condon.

Statistical interpretation of various formulations of quantum mechanics, by Professor J. H. Van Vleck. Discussion led by Professors E. H. Kennard, D. J. Struik, and E. C. Kemble.

The problem of symmetry in quantum mechanics, by Professor Hermann Weyl. Discussion led by Professors G. Y. Rainich, S. A. Goudsmit, and H. W. Brinkmann.

Harmonic analysis and quantum mechanics, by Professor Norbert Wiener. Discussion led by Dr. T. H. Gronwall and Professors H. P. Robertson and G. E. Uhlenbeck.

More than 400 persons, a much larger number than had been anticipated, including chemists as well as mathematicians and physicists, were present at the sessions of this symposium.

Titles and abstracts of the papers read at the regular sessions of the Society follow below. The presiding officers were as follows: Vice-President Kellogg over Section I (Analysis) and (relieved by Professor Lefschetz) over Section III (Analysis and Applications); Vice-President Young over Section II (Geometry) and Section IV (Algebra and Analysis Situs); ex-President Van Vleck at the general session on Friday morning; Professor R. C. Archibald at the joint session with the Mathematical Association and Section A; ex-President Birkhoff during the Gibbs Lecture and (relieved by Dr. T. C. Fry) at the afternoon session of the Symposium on Quantum Mechanics; Professor E. V. Huntington at the joint session with Section $\mathrm{K}$; and Professor K. T. Compton at the morning session of the Symposium. The papers numbered 1-21 were read before Section 
I, Nos. 22-39 before Section II, Nos. 40-63 before Section III, Nos. 64-92 before Section IV, No. 93 at the general session, and Nos. 94-99 at the joint session with Section K. The papers of the following authors were read by title: Albert (first, second, and fourth papers), Altshiller-Court (second paper), Ayres, Barrow, Berry, Brinkmann (second and third papers), Douglas, Ettlinger, Frink (third paper), Garver, Harkin, Hickey, Keller, Littauer, Lubben (first paper), McGiffert, Poritsky (last three papers), Powers, Reynolds, Roberts, Rutledge, Schwatt, Sheffer (first paper), Stone (first three papers), Tamarkin (first and second papers), Sullivan, G. T. Whyburn, W. M. Whyburn, and Widder. Professor Hagihara was introduced by Professor Birkhoff, Mr. Muckenhoupt by Professor Wiener, and Dr. Vasilesco by Professor Kellogg.

1. Professor H. J. Ettlinger: Expansion problems for general second-order systems.

Expansion problems are considered for the system $y^{\prime}=K(x, n) z, z^{\prime}$ $=G(x, n) y$, with self-adjoint boundary conditions. The method used is that of transforming to polar coordinates, $y=u \cos v, z=u \sin v$. Asymptotic expansions are obtained for characteristic functions and characteristic numbers. Expansion theorems are obtained for a pair of arbitrary functions. The results contain the work of Camp, Hurwitz, and others as special cases.

2. Dr. I. M. Sheffer (National Research Fellow): On the properties of systems of polynomials which are functionally orthogonal.

Let there be given a linear operator $L$ which assigns a number to each member $u(x)$ of a suitable class of functions defined over a certain range $R$ of points: $L[u(x)]=c$. Two simple examples of $L$ are (1) $L[u]$ $=\int_{a}^{b} p(x) u(x) d x$, where $p(x)$ is a given function; (2) $L[u]=\sum_{k=0}^{\infty} p_{k} u\left(x_{k}\right)$, where the sequence $\left\{p_{k}\right\}$ is given. In (1) the range $R$ is the set of points $a \leqq x \leqq b$; in (2) $R$ is the set of points $x_{0}, x_{1}, x_{2}, \cdots$. The functions $u(x)$ and $v(x)$ are said to be $L$-orthogonal if $L[u(x) \cdot v(x)]=0$. We consider a system of polynomials $\left\{T_{n}(x)\right\}$ such that $L\left[T_{m}(x) T_{n}(x)\right]=0, m \neq n$, and examine the properties of this system. These properties are found to be essentially inherent in the linear character of the operator.

3. Dr. I. M. Sheffer: On systems of polynomials which are permutable.

The Appell polynomials have the property of permutability: that is, if $\left\{P_{n}(x)\right\},\left\{Q_{n}(x)\right\}$ are any two sets of Appell polynomials, they are 
permutable. Appell polynomials form, then, a class of permutable sets of polynomials. But this is not the only class having this property. We obtain further such classes, and examine the general theory. To each set of polynomials we assign a characteristic equation which the set satisfies. The theory of permutability links up, on one side, with the theory of summability by the method of Toeplitz and Hurwitz-Silverman, and, on the other side, with the condition that certain linear operators be themselves permutable.

4. Dr. Irwin Roman: A formula for the summation of series.

Taylor's theorem leads to the formula $f(n)=Z(n+h)-Z(n-h)$, where $Z(x)=(2 h)^{-1}\left[\int f(x) d x+\sum_{u=1}^{\infty} b_{2 u} h^{2 u} f^{(2 u-1)}(x)\right]$. Hence we may write the formula $\sum_{n=t}^{s} f(k+2 h n)=Z[k+(2 s+1) h]-Z[k+(2 t-1) h]$, and also the formula $\int_{n-n}^{n+h} f(x) d x=2 h f(n)-\sum_{u=1}^{\infty} b_{2 u} h^{2 u}\left[f^{(2 u-1)}(n+h)-f^{(2 u-1)}(n-h)\right]$. The constants $b_{2 u}$ are determined by a recursion formula. They are expressible in terms of the Bernoulli numbers and conversely. Illustrative examples indicate the wide applicability of the formulas, but no attempt is made to discuss the conditions necessary for the formulas to be applicable to a particular problem.

\section{Professor J. D. Tamarkin: On Volterra-Stieltjes integral equations.}

In this paper a system of $n$ integral equations is considered of the type $Y(x)=F(x)+\int_{a}^{x} d_{\xi} K(x, \xi) Y(\xi)$, where (in matrix notation) the vector $F(x)$ and the matrix $K(x, \xi)$ are given. It is assumed that $F(x)$ is bounded and Borel measurable and $K(x, \xi)$ is bounded on $a \leqq \xi \leqq x \leqq b$, Borel measurable in $x$ for fixed $\xi$ and continuous and of bounded variation in $\xi$ for fixed $x$, the total variation being uniformly bounded. The uniqueness and existence of a solution (bounded and Borel measurable) is proved. Certain boundary problems of Volterra-Stieltjes type are considered, and the notion of the Green's function for such problems introduced. The results obtained are applied to the discussion of the generalized adjoint problem.

6. Professor J. D. Tamarkin: Expansion of an arbitrary function in fundamental functions of a boundary value problem of integral type.

It is shown, by means of some results previously obtained by the author, that if the "boundary" conditions contain integral terms, then the set of fundamental functions in general is not closed, and a set of supplementary conditions must be satisfied by the function which has to be expanded. However, the set of fundamental functions can always be extended to a closed one by addition of a finite number of functions which are easily determined.

\section{Professors Einar Hille and J. D. Tamarkin: On the sum-} mability of Fourier series. Note 2.

The authors have continued their work on the summability of Fourier series and have found sufficient conditions for the applicability of Hausdorff 
means for almost all values of the variable. A more detailed outline of the results will appear in a forthcoming number of the Proceedings of the National Academy of Sciences.

\section{Dr. F. W. Perkins: On the theory of mediation.}

By the mediant of a function $F_{0}(P)$ (where $P$ is a point of a bounded open region $T$ in a 3 -dimensional euclidean space) is meant the function $F_{1}(P)$ whose value at $P$ is equal to the average value of $F_{0}(P)$ on the surface of the largest sphere with center at $P$ containing in its interior only interior points of $T$. Another form of mediation may be obtained by using the average with respect to volume. The definition may also be generalized for a region $T$ in an $n$-dimensional euclidean space. In this paper a number of general properties of mediation are obtained, and especial attention is given to the discussion of the sequence $F_{0}(P), F_{1}(P), F_{2}(P), \cdots$, obtained by successive application of the process. The study of this sequence shows that mediation is closely connected with the theory of harmonic functions, particularly with the first boundary value problem of potential theory. This phase of the theory has been investigated by Lebesgue (Comptes Rendus, vol. 154 (1912), p. 355), and the present author (Comptes Rendus, vol. 184 (1927), p. 182).

9. Professor I. J. Schwatt: The summation of series the terms of which alternate in sign, not term-by-term but by groups of terms.

The author has considered the sum of like powers of the first $n$ natural numbers, the terms alternating in sign by groups of terms, so that, say, $h$ positive terms are followed by $h$ negative terms, and also the case where $\mathrm{t} h$ numbers of terms in the positive and negative groups are unequal. The results involve the Bernoulli numbers.

10. Professor I. A. Barnett: A characteristic property of conformal transformations in function space.

Let $R_{x}$ denote the totality of all continuous functions $f(x)$; and $R_{x}^{\prime}$ the totality of all functions $[f(x), \sigma]$, where $\sigma$ is a real continuous variable. The projective group in the space $R_{x}^{\prime}$ is defined and explicit formulas obtained for all the infinitesimal transformations in this space. The author then finds the subgroup all of whose transformations leave the manifold $\int f^{2}(x) d x+\sigma^{2}=1$ invariant. Calling these transformations $S$, he proves that if $S$ are subjected to a stereographic projection in the space $R_{x}$ on the linear spread $\sigma=$ const., the resulting transformations are the same as those that leave invariant the angle between any two curves in $R_{x}$, viz., the conformal group in $R_{x}$. This is a generalization of a well known property in spaces of a finite number of dimensions.

11. Professor M. H. Stone: Expansions associated with self-adjoint elliptic partial differential equations.

In the present paper the classical problem of the flow of heat in a nonhomogeneous conducting medium with assigned initial temperature and 
permanently assigned boundary temperature is considered from a modern point of view. The solution of the differential equation of parabolic type governing the flow is studied as a functional of the boundary conditions. There results an important theorem concerning the representation of an arbitrary bounded measurable function in terms of the solutions of an elliptic partial differential equation involving a parameter.

12. Professor M. H. Stone: Theory of Hermitian transforms.

In a previous communication to the Society, the author has considered the general theory of Hermitian symmetric operators in abstract Hilbert space. Operators of this type which satisfy the condition that some iterate is the identity are studied in the present paper. It is found that if $T$ is such an operator either $T$ or $T^{2}$ is the identity. The second alternative is given special consideration. A useful sufficient condition that an operator be of this character is obtained, and is then applied to yield a unified treatment of certain familiar operators of analysis, including the Fourier and Hankel transforms.

\section{Professor M. H. Stone: Gauss' law of error in several} variables.

In the Mathematische Zeitschrift for 1923, Pólya gave a derivation of Gauss' law of error from a functional equation. The present paper extends the derivation to the law of error for several variables. The method is similar to that of Pólya, and the results differ very little from his. With the increase of the number of variables from one to several, it is necessary to increase the number of hypotheses concerning the law of error in order to determine it uniquely. The relation of these hypotheses to the classical assumptions of Laplace and of Gauss is discussed.

14. Professor M. H. Stone: Unitary transformations in abstract Hilbert space.

In this paper the following theorem is proved: every one-parameter continuous group of unitary transformations in abstract Hilbert space possesses a generating infinitesimal transformation of the form $i T$, where $T$ is an Hermitian symmetric operator which is maximal, and conversely.

15. Professor G. M. Robison: The consistency and equivalence of certain definitions of summability of divergent double series.

This paper deals with definitions of summability of divergent double sequences which can be expressed by means of a linear transformation. Let $x_{m, n}$ be the given sequence. A new sequence $y_{m, n}$ is defined by the relation $y_{m, n}=\sum_{k=1, l=1}^{m, n} a_{m, n, k, l} x_{k, l}$, where the $a_{m, n, k, l}$ 's form a set of constants defining the transformation, which we denote by $(A)$. If $\lim _{m, n \rightarrow \infty} y_{m, n}$ exists and equals $\lambda$, we say that $\lambda$ is the generalized value of the sequence $\left(x_{m, n}\right)$ by the transformation $(A)$. This paper shows that all regular transformations of a certain class are consistent and gives a criterion for the equivalence of two regular definitions of summability. 
16. Dr. Stefan Serghiesco: On the number of multiple common roots of a system of $n$ equations.

In several earlier papers, the author has introduced a new method for obtaining the number of simple common zeros of a set of $n$ functions, and has given two new general formulas. In the present paper, the author extends this method of the case of multiple common zeros, stating the conditions of possibility with the corresponding formulas.

17. Dr. T. H. Rawles: On minimizing a function of two definite integrals.

The problem discussed in this paper is that of minimizing a function of two definite integrals of the form $U\left(\int_{x_{1}}^{x_{2}} f\left(x, y, y^{\prime}\right) d x, \int_{x_{1}}^{x_{2}} g\left(x, y, y^{\prime}\right) d x\right)$. Besides obtaining the extremals, further necessary conditions are developed analogous to those of Legendre and Jacobi in the simplest problem in the calculus of variations. Sufficient conditions are obtained by means of an invariant integral.

18. Professor George Rutledge: Limiting values of Lagrangean coefficients.

The coefficients, $C_{j}$, of the Lagrange polynomial, of degree $2 n$, $P^{[2 n]}(\lambda)=C_{-n} y_{-n}+\cdots+C_{0} y_{0}+\cdots+C_{n} y_{n}$, useful for interpolation without differences, approach limiting values as $n$ becomes infinite, as follows: $\lim C_{j}=(-1)^{j+1}[\lambda /(j-\lambda)](\sin \lambda \pi) /(\lambda \pi)$.

19. Professor George Rutledge: Fundamental tables for Lagrangean coefficients.

This paper gives tables with fifteen decimal places from which the coefficients of the Lagrange polynomial are readily derived for any range of $\lambda$ and for any even degree up to and including sixteen.

20. Professor D. F. Barrow: Functions of iteration and their relation to non-linear difference equations.

The symbol $f^{n}(t)$ denotes $n$ iterations of the given function $f(t)$, and defines a function of two arguments; $t$, a continuous variable, and $n$, an integer. The author proposes to define this function of iteration for all values of $n$; and this is, by definition, made tantamount to finding analytic solutions of the difference equation $\phi(x+1)=f[\phi(x)]$. The solution of the problem is embodied in a group of existence theorems and approximation formulas for numerical computation. The chief interest centers about the points of equilibrium which satisfy $A=f(A)$. The paper closes with a number of examples, including the analytic iteration of the sine and exponential functions, that is, the solution of the equations $\phi(x+1)=e^{\phi(x)}$, and $\phi(x+1)=\sin \phi(x)$.

21. Professor D. V. Widder: An inequality related to one of Hilbert. 
In the Mathematische Zeitschrift of 1921, L. Fejér and F. Riesz established a fundamental inequality, and applied it to the proof of a familiar inequality of Hilbert. They proved that if $f(z)$ is analytic in a circle $|z| \leqq 1$, then $\int_{-1}^{1}|f(z)|^{2} d z \leqq \frac{1}{2} \int_{-\pi}^{\pi}\left|f\left(\epsilon^{i \theta}\right)\right|^{2} d \theta$. In the present paper, the circle. is replaced by a half plane, and then the inequality $\int_{0}^{\infty}|f(z)|^{2} d z \leqq$ $\frac{1}{2} \int_{-\infty}^{\infty}|f(i y)|{ }^{2} d y$ is established under suitable conditions. This result is used to prove the inequality $\sum_{m, n=0}^{n} a_{m} a_{n} /(m+n+1) \leqq \pi \sum_{m, n=0}^{N}(m+n) ! a_{m} a_{n}$ $\cdot\left(m ! n ! 2^{m+n+1}\right)^{-1}$, where the $a_{m}$ are any real numbers. This inequality includes that of Hiltert, for it is shown that the right hand member is not greater than $\pi \sum_{n-0}^{N} a_{n}^{2}$.

\section{Professor C. A. Nelson: On surfaces possessing a net} of plane isothermally-conjugate curves.

It is well known that the Laplace transforms of a surface relative to a conjugate system of plane curves are developable. This paper studies the effect of the isothermal property upon such surfaces and their projective properties are determined. The problem of integrating the defining system of partial differential equations is investigated, and, in certain cases, the complete integration is effected, so that explicit formulas are obtained for the coordinates of the surface.

\section{Professor C. T. Sullivan: The determination of plane} nets characterized by certain properties of their Laplace transforms.

This paper will appear in full in an early issue of this Bulletin.

\section{Dr. M. M. Slotnick: A conjugate net of special type.}

A conjugate net for which each tangent to the $v$-curve of its first Laplace transform intersects the tangent to the corresponding $u$-curve of its minus first Laplace transform will be called a net $\Delta$. The point of intersection of these tangents is the pivotal point of the axis. A net whose ray curves or whose axis curves are indeterminate is a net $\Delta$. A characteristic property of nets $\Delta$ is the following: the tangents to the axis curves and to the ray curves separate the tangents to the curves of the net harmonically. If a net $\Delta$ has equal point [tangential] invariants, the ray [axis] curves constitute the associate conjugate net of the given net.

\section{Professor Edward Kasner: Higher partial derivatives of polygenic functions of several variables.}

The higher partial derivatives of polygenic functions of two or more complex variables depend, not only on the curves of approach for each of the independent variables (slope, curvature, etc.), but also on the correspondence between the curves. Also, as indicated in earlier papers (see Transactions of this Society, vol. 30, p. 818) we must take into account the order of the variables. 


\section{Professor T. R. Hollcroft: The variety of order $n$ in $n$ dimensions.}

There is a one-to-one correspondence between varieties of order $n$ in $i$ dimensions and varieties of order $i$ in $n$ dimensions, since the number of conditions necessary to determine each is the same. In this correspondence, the variety for which $n=i$ is self corresponding. It is proved that every general variety of order $n$ in $n$ dimensions, $n \geqq 3$, contains the following: an $(n-3)$-fold infinity of lines; a triple infinity of plane elliptic cubics; a single infinity of conics; a finite number of sets of three coplanar lines; for $n \geqq 4$, a $(d+1)(d-3)$-fold infinity of sets of $d+1$ lines, each set contained in a linear $d$-space, $d<n$; an $(n-1)$-fold infinity of space cubics; a triple infinity of rational space quartics; a fourfold infinity of elliptic space quartics. The general variety of order four in four dimensions contains a finite number of sets of four coplanar lines, and a finite number of sets of four non-intersecting lines, each latter set contained in a linear three-space. The number of these sets is found.

\section{Dr. Oscar Zariski: The existence problem for algebraic} functions of two variables.

The determination of the algebraic functions of two complex variables $x, y$, which possess a given branch curve $D$ ( a two-dimensional Riemann surface) in the real space $S_{4}$ of the variables $x$ and $y$, depends upon the determination of the fundamental group $G$ of the residual space $S_{4}-D$. The following theorems are stated: (1) If $D$, of order $n$, is irreducible and possesses double nodal points only, then $G$ is the cyclic group of order $n$. (2) If the order $n$ of the irreducible curve $D$ is $<6$, then $G$ is always the cyclic group of order $n$. (3) The first example of non-cyclic fundamental group is furnished by sextics with 6 cusps on a conic. Here $G$ possesses two generators $g_{1}, g_{2}$, the generating relations being $g_{1} g_{2} g_{1}=g_{2} g_{1} g_{2},\left(g_{1} g_{2} g_{1}\right)^{2}=1$. (4) If $D$ possesses double nodal points only, and breaks up into $k$ curves of order $n_{1}, n_{2}, \cdots, n_{k}$, respectively, then $G$ is an abelian group generated by

$k$ generators $g_{1}, g_{2}, \cdots, g_{k}$, subjected to one generating relation: $g_{1}{ }^{\rho}=1$, where $\rho$ is the h.c.d. of $n_{1}, n_{2}, \cdots, n_{k}$. The following questions are raised: (a) What is the minimum number of cusps which an irreducible curve of given order and genus must possess, in order that $G$ should not be cyclic? (b) Does the fundamental group depend upon the number of the cusps only, or does it also depend upon the position of the cusps?

\section{Professor Malcolm Foster: Note on cyclic systems.}

It is shown in this paper that the four focal surfaces of a cyclic system whose planes envelope a non-developable surface are the envelopes of the two families of spheres which contain the circles of the system and have their centers at the focal points of the associated cyclic congruence, and that the focal planes of this cyclic congruence bisect the arcs between corresponding focal points. If the planes of the circles of the system envelope the unit sphere, the associated cyclic congruence is normal; and the circles under an inversion go over into a second family of circles whose axes also 
constitute a normal congruence. The paper also considers various cyclic systems lying in the $X Z$-plane of the moving trihedral of the surface of reference.

\section{Mr. H. L. Dorwart: The discussion of a construction of} the rational plane quintic.

The rational plane quintic, $R_{2}{ }^{5}$, has a perspective conic, $C$, and if 2 of the 6 nodes of the $R_{2}{ }^{5}$ are in a quadratic involution, it has 15 involution contact conics. The pairs in the involution are given by the extra intersections of conics on the remaining 4 nodes. Two of these conics will touch $R_{2}{ }^{5}$, and will have a line, $L$, in common with $C$. Since $L$ is perspective with $C$, and since each conic on the 4 nodes cuts out 2 points on $L$ which are in $(1,1)$ correspondence with the extra 2 intersections of the conic and $R_{2}{ }^{5}$, this gives a method for constructing the curve; it is mentioned by Morley in The contact conics of the plane quintic curve (The Johns Hopkins Circular, 1912). If two of the four nodes are at $I$ and $J$, the pencil of conics is a pencil of circles, and the construction is simplified. We show a number of quintics, obtained by taking the different conic sections for the perspective conic, and by varying the relative positions of conic, line, and nodes.

\section{Professor C. A. Rupp: A geometric interpretation of linear dependence in $n$-space.}

We consider the geometrical implications of the algebraic statement that the set of homogeneous Grassmann-Plucker coordinates of an $S_{k}$ in $S_{n}$ is a linear combination of the sets of coordinates of several $S_{k}$ 's in the same $S_{n}$. The author has previously shown that $n+1$ lines (or $S_{n-2}$ 's) which are linearly dependent are in the position of Schläfli, but here gives a simpler proof. A set of Schlafli or linearly dependent spaces determine and lie upon a variety of order and dimension $n-1$; Segre discussed this variety synthetically, as does Wong in a recent paper. By using the concept of linear dependence as central in the study of this variety, the author has found a method of obtaining its equation, and displays several examples in spaces of low dimensions. Another of Segre's synthetic discoveries was that of systems of "associated" lines and planes in $S_{4}$ and $S_{5}$. They are examples of a more general type of configuration, consisting of linearly dependent flat spaces; several of these configurations are discussed, and in particular it is shown how many spaces of such a set may be chosen arbitrarily.

31. Professor Nathan Altshiller-Court: On nodal cubics circumscribed about a triangle. First paper.

Consider the pencil of nodal cubics $\Sigma$ circumscribed about the triangle $A_{1} A_{2} A_{3}$ and having in common the double point $O$ and the two tangents at this point (see this Bulletin, vol. 26, pp. 203 and 114). Any point $P_{1, n}$ of the side $a_{1} \equiv A_{2} A_{3}$ of the triangle $A_{1} A_{2} A_{3}$ determines a cubic $\Sigma_{n}$ of the pencil $\Sigma$. (1) As the point $P_{1, n}$ describes the line $a_{1}$ the tangents to the respective cubics envelope a tricuspidal quartic $\left(\Delta_{1}\right)$ tangent to $a_{1}$ in $A_{2}$ and $A_{3}$, and to the line $O A_{1}$ in the points $A_{1}$. (2) The tangents drawn from $P_{1, n}$ to the respective cubics pass through two fixed points, namely the 
points of intersection of the line $O A_{1}$ with $\left(\Delta_{1}\right)$. (3) The points of contact of these tangents lie on two fixed straight lines passing through $O$. (4) The line joining the points of contact of these two tangents passes through a fixed point. (5) The locus of the tangential of $P_{1, n}$ is a quartic curve circumscribed about the triangle $A_{1} A_{2} A_{3}$ and having a triple point at $O$. (6) The locus of the corresponding point of $P_{1, n}$ is a conic passing through the points $O, A_{1}, A_{2}, A_{3}$. (7) The line joining $P_{1, n}$ to its corresponding point envelopes a tricuspidal quartic, the line $a_{1}$ being doubly tangent to the curve.

\section{Professor Nathan Altshiller-Court: The Longchamps} sphere. A definition.

The usual definition of the Longchamps circle of a triangle (American Mathematical Monthly, 1926, p. 368) may be modified as follows (Mathesis, 1928, p. 156): Consider the three circles each having for center the vertex of a triangle and for the square of its radius the sum of the powers of the remaining two vertices with respect to the conjugate circle of the triangle. The orthogonal circle of these three circles is, by definition, the Longchamps circle of the triangle. This new form of the definition suggests an immediately obvious extension to space. It is shown that the sphere so defined has another characteristic property, analogous to one of the circle, namely, that it is the conjugate sphere of the anticomplementary tetrahedron of the given tetrahedron.

\section{Professor A. D. Campbell: Pencils of quadrics in the Galois fields of order $2^{n}$. \\ In the Galois fields of order $2^{n}$ the quadric $Q \equiv a x^{2}+b y^{2}+c z^{2}+d w^{2}$ $+f y z+g z x+h x y+l x w+m y w+n z w=0$ has the discriminant $\Delta \equiv f^{2} l^{2}+g^{2} m^{2}$ $+h^{2} n^{2} \equiv(f l+g m+h n)^{2}$. Hence the discriminant $\Delta$ of a pencil of quadries $P \equiv 2 Q_{1}+\mu Q_{2}=0$ shows that $P$ has one or two degenerate quadrics if $\Delta \not \equiv 0$, but if $\Delta \equiv 0, P$ consists entirely of degenerate quadrics. In this paper are derived the classes of pencils of quadrics in the Galois fields of order $2^{n}$ and a typical pencil is given for each class. There occur pencils without degenerate quadrics. The most difficult but most interesting part of the problem is the discussion of the equivalence or nonequivalence of typical pencils.}

\section{Professor C. N. Reynolds: On polyhedra.}

Given a finite set of integers, $a_{n}(n \geqq 3)$, when is it possible to construct a convex simply connected closed polyhedron bounded by $a_{n} n$-sided polygons with three polygons meeting at each vertex? It is known that Euler's theorem implies the necessity of the condition (1): $\Sigma_{3}(n-6) a_{n}$ $+12=0$. This equation has 610 solutions for which $a \equiv \Sigma a_{n}<11$, whereas only 140 of them are characteristic of known polyhedra. The most important propositions of the present paper yield a group of inequalities of the form (2): $\Sigma_{3}\left(n^{2}+p n+q\right) a_{n} \leqq f(a)$, which eliminate 347 of the 470 "extraneous solutions" of (1) mentioned above. As $a$ increases beyond ten, the number of solutions of (1) eliminated by (2) increases numerically, but the 
ratio of this number to the total number of extraneous solutions of (1) decreases.

\section{Professor C. N. Reynolds: On the problem of coloring} maps in four colors. Third paper.

Having considered (Annals of Mathematics, (2), vol. 28, pp. 1, 447) the geometry of maps which are irreducible with respect to reductions dealing with the immediate neighborhoods of regions, the author in the present paper deals with the gross structure of irreducible maps. The results of the preceding paper on polyhedra are found to be of value in describing such maps.

\section{Miss Deborah M. Hickey: Isometric circles on the} sphere.

Let the surface of the sphere be transformed into itself in a one-to-one and directly conformal manner. In this paper it is proved that, except for a rotation of the sphere, the areas of magnification and of diminution constitute respectively the smaller and larger spherical caps formed by a small circle on the sphere. Certain of the properties of this isometric circle are derived.

\section{Dr. Jesse Douglas (National Research Fellow): On} the problem of Lagrange in the calculus of variations.

The simplest form of this problem is to minimize the integral $\int F\left(x, y, z, y^{\prime}, z^{\prime}\right) d x$ under the auxiliary condition $\phi\left(x, y, z, y^{\prime}, z^{\prime}\right)=0$. A singular or so-called anormal case arises of which various interpretations have been given by Mayer (Mathematische Annalen, vol. 26 (1886)), von Escherich (Wiener Sitzungsberichte, vols. 107 (1898), 108 (1899), 110 (1901)), and Hahn (Mathematische Annalen, vol. 58 (1903-04)). The principal result of the present paper is a new interpretation of the anormal case to the effect that the characteristics of the auxiliary Monge equation are then extremals. Various other aspects of the problem are considered.

38. Dr. Jesse Douglas: The affine, voluminar, and descriptive equivalence of spaces of $K$-spreads.

(See the author's abstract The geometry of systems of $K$-spreads, this Bulletin, Jan.-Feb., 1928.) Two spaces of $K$-spreads are (1) affinely equivalent if they can be converted into one another by an arbitrary analytic transformation of coordinates, combined with a linear transformation of parameters, (2) voluminarly equivalent if they can be converted into one another by an arbitrary analytic transformation of coordinates combined with a transformation of parameters with constant jacobian, (3) descriptively equivalent if they can be converted into one another by an arbitrary analytic transformation of coordinates combined with an arbitrary analytic transformation of parameters. Necessary and sufficient conditions are established for the three types of equivalence. In particular, conditions are derived for affine, voluminar, and descriptive flatness, that is, for the 
respective types of equivalence to the space $\partial^{2} x^{i} / \partial u^{\alpha} \partial u^{\beta}=0$. The methods and results are in the order of ideas of Christoffel's criteria for the equivalence of two quadratic differential forms, the criteria of Veblen and J. M. Thomas (Annals of Mathematics, Dec., 1925) for the projective (descriptive) equivalence of two restricted spaces of paths, and the author's criteria (abstract in this Bulletin, July-Aug., 1928) for the affine and descriptive equivalence of two general spaces of paths.

39. Dr. Jesse Douglas: The Weyl tensor in the general geometry of paths.

In the author's paper The general geometry of paths (Annals of Mathematics, (2), vol. 29, No. 2) a formula (7.11) is given for the Weyl tensor, analogous to the one given by J. M. Thomas (Proceedings of the National Academy of Sciences, vol. 11, pp. 207-209) for the restricted geometry of paths. In the present note a second formula is established for the Weyl tensor like the one given for the restricted theory by Veblen and T. Y. Thomas (Transactions of this Society, vol. 25, p. 560), being based on considering the effect on the curvature tensor of an arbitrary change of parameter on the paths.

40. Dr. Morris Marden (National Research Fellow): On the zeros of linear partial fractions.

This paper determines the locus of the zeros of the partial fraction $F=\sum_{1}^{p} m_{j} /\left(z-z_{j}\right)$, when each $z_{j}$ varies over a given circular region $C_{j}$. Its results are applied to the location of the zeros of the derivative of a rational function, especially of a polynomial, to the location of the zeros of the Jacobian of two binary forms, and to a certain cross-ratio problem. Its theorems are essentially generalizations obtained analytically of theorems previously obtained by Lucas, Bôcher, and Walsh.

41. Professor W. W. Elliott: Note on generalized Green's functions.

In a previous paper (American Journal of Mathematics, vol. 50, p. 243) the existence of generalized Green's functions for compatible differential systems has been demonstrated, and the characteristic properties of certain types of symmetrical generalized Green's functions have been studied. In the present paper other types of symmetrical generalized Green's functions are discussed.

\section{Dr. W. J. Trjitzinsky: On quasi-analytic functions.}

Borel's monogenic functions, whose discovery antedates that of quasianalytic functions of a real variable (Denjoy), are quasi-analytic in a complex domain $C$ from which a point set of measure zero has been excluded. In this paper it is shown that Borel's functions belong to the first Denjoy class with certain restrictions on the domain of existence. Further, series of the form $\sum c_{n} /\left(x-a_{n}\right)$, which are quasi-analytic when the $c_{n}$ satisfy conditions given by Borel and Carleman, are considered. With less re- 
strictive specifications of the $c_{n}$, conditions are found under which such series belong to the first Denjoy class. Carleman's representation when initial values are given is extended. Necessary and sufficient conditions are found securing convergence of a sequence of polynomials to a quasianalytic function. A new representation of a class of functions is given.

\section{Professor R. L. Jeffery: The uniform approximation} by means of Riemann sums of a function defined by a Lebesgue integral.

Let $f(x, y)$ defined on a bounded domain be summable in $x$ for each $y$. The chief concern of this paper is to determine conditions on $f(x, y)$ under which $\int_{a}^{b} f(x, y) d x$ can be approximated by a Riemann sum $\sum f\left(\xi_{i}, y\right)\left(x_{i}-x_{i-1}\right)$ uniformly in $y$. Extending a result due to Lebesgue, concerning the simultaneous approximation of $p$ functions $g_{1}(x), \cdots, g_{p}(x)$ (Annales de Toulouse, (3), vol. 1, p. 33), the author shows that if $f(x, y)$ is bounded and continuous in $y$ for each $x$, then for any subdivision with norm sufficiently small, there exists $\xi_{i}$ on $\left(x_{i-1}, x_{i}\right)$ such that $\mid \int_{a}^{b} f(x, y) d x$ $-\sum f\left(\xi_{i}, y\right)\left(x_{i}-x_{i-1}\right) \mid<\epsilon$, independent of $y$. Lebesgue remarks that his result holds for an infinite sequence $g_{1}(x), g_{2}(x), \ldots$. It seems that this statement needs some qualification, even when $g_{n}(x)$ is bounded in $x$ and $n$.

44. Dr. Hillel Poritsky (National Research Fellow): The billiard-ball problem on a convex table.

The problem considered is the motion of a point in the plane inside a simple closed convex curve in a straight line, with reflection at the boundary so that the angle of incidence is equal to the angle of reflection. This problem, because of its lack of formal complication, is well suited for illustrating various methods in dynamics, and has been used by Birkhoff in connection with Poincaré's geometric theorem and his own extension of it (Acta Mathematica, vol. 50). The results obtained in the present paper are based largely on the existence of an invariant integral for the transformation associated with the reflection of paths at the boundary. Thus, the existence and distribution of certain periodic orbits as well as of orbits passing through two points or satisfying other conditions is inferred directly from the existence of the invariant integral, by simple geometric methods. The integrability of the problem is studied from various points of view leading to results of interest in projective and differential geometry. Morse's theory of extremals is found to apply, in spite of the discontinuity in the calculus of variations problem whose extremals constitute the above orbits.

\section{Dr. Hillel Poritsky: Cauchy-Green expansions.}

Under this title are considered certain expansions of analytic functions over points $P$ inside a surface $S$, that express $u$ as a sum of integrals over $S$ involving the boundary values of $u, \nabla^{2} u, \nabla^{4} u, \cdots$, multiplied by certain simple functions of the distance from $P$ and its normal derivative. These expansions form the analogue, for analytic functions of several variables, of the Cauchy integral for a function of a complex variable, and, more 
precisely, of the Green "double-layer" integral for harmonic functions. A finite series of this type with a remainder is obtained by successive application of Green's theorem. It is then shown that if the number of terms be allowed to become infinite the remainder, under proper restrictions on $S$, will approach zero. The restrictions on $S$ may be materially lightened if $u$ satisfies certain partial differential equations.

\section{Dr. Hillel Poritsky: On certain integrals over spheres.}

It is shown that the linear operation on a function $u$ that consists in replacing $u$ over each of a family of concentric spherical surfaces by the function of the radius $\int u d S / \int d S$ or the "average" of $u$ over the spherical surface is permutable with the Laplacian operator. This property is further extended to certain other operators that may be described as leading from $u$ to the various terms in the expansion of $u$ in a series of spherical surface harmonics. From these simple facts are deduced a great many results, some familiar, some new, in the theory of harmonic and related functions. Among these are various laws of the spherical mean, integral representations for spherical harmonics and Bessel functions, and the reduction of certain expansions to Fourier expansions. The motif of the paper is geometric throughout.

\section{Dr. Hillel Poritsky: On certain oscillation theorems.}

It is shown that a homogeneous harmonic polynomial of degree $n$ that does not vanish along a certain ray, $l$, through the origin, must change sign within the conical region about $l$ in which the harmonic polynomial $p_{n}=r^{n} P_{n}(\cos \theta)$ stays positive, where $r$ is the distance from the origin and $\theta$ is the angle that rays through the origin form with $l$. Analogous results are established for bi-harmonic, tri-harmonic, $\cdots$, polynomials, as well as for solutions of certain partial differential equations. In this connection a necessary and sufficient condition is derived and utilized for the existence of a linear combination of several given functions of a single variable that does not vanish throughout a certain interval. It is then shown how some of the former results may be extended to regions of arbitrary shape by the use of the characteristic parameter values associated with these regions.

48. Professor E. R. Hedrick: Integrals along given curves in the complex domain.

In this paper the author discusses integrals taken along given curves in the complex domain, for functions that are non-analytic. It is shown that certain types of theorems are easily extended from the traditional theory to include integrals of non-analytic functions whenever the paths are fixed in advance. Thus the theorems recently stated by Pfeiffer (this Bulletin, vol. 34 (1928), pp. 656-664) can be thus extended.

49. Professor Marston Morse: A generalization of Sturm's separation theorem in $n$ dimensions.

The author considers a set of $n$ self-adjoint, second-order, linear, homogeneous differential equations in $n$ dependent variables $(y)$ and an in- 
dependent variable $x$. The matrix of the coefficients of the second derivatives is assumed to have a characteristic determinant all of whose roots are positive. Any such system of differential equations are shown to be the Jacobi differential equations of an easily determined integral in the calculus of variations. With this point of view, the author considers the determinants $D(x)$ of systems of $n$ linearly independent solutions which are mutually conjugate in the sense of von Escherich. He shows that if one of these determinants vanishes $n+r$ times on a closed interval of the $x$ axis, any other of these determinants will vanish at least $r$ times on the same closed interval. This theorem and others appeared as corollaries of a fundamental theorem on the type number of a quadratic form discovered and used in a development of the calculus of variations in the large.

\section{Professor J. L. Walsh: On approximation by rational} functions to an arbitrary function of a complex variable.

In the plane of the complex variable $z$, let $M$ be a closed point set whose boundary consists of a finite number of Jordan arcs which do not separate the plane into more than a finite number of regions. Then an arbitrary function $f(z)$, continuous on $M$ and analytic in the interior points of $M$ can be uniformly approximated on $M$ as closely as desired by a rational function of $z$.

\section{Professor Orrin Frink: Functions defined over an} abstract set.

In this paper the theorems that a function continuous over a connected set assumes all values between its maximum and minimum, and that a function continuous over a compact-in-itself set is bounded and assumes its upper and lower bounds, the Heine-Borel theorem, and other standard theorems of real function theory, are proved either for the general topological space or for a space subject to one weak postulate. The theorems are more general than the corresponding ones proved by Fréchet for neighborhood spaces.

\section{Professor James McGiffert: Two closed forms of} particular solutions of the linear differential equation of second order $\left(A+B x^{2}\right) y^{\prime \prime}+C x y^{\prime}+D y=0$.

We assume a solution in series form, $y=\sum_{m=0}^{\infty} a_{m} x^{m}$. Substituting this value for $y$, and its two derivatives for $y^{\prime}$ and $y^{\prime \prime}$, and equating the com. bined coefficients of $x^{m}$ to zero, we obtain the recurrence relation $a_{m+2}=-(B / A)\left[\left(m^{2}+(C-B) m / B+D / B\right) /((m+1)(m+2))\right] a_{m}$. This rather complicated recurrence relation does not in general lead to useful forms of particular solutions. We may, however, so choose the coefficients, that the numerator of the fraction in brackets shall have $m+1$ or $m+2$ as a factor. Then the recurrence relation will be much simpler, and will reduce to two binomial forms, depending on the value of $D$ in terms of $B$ and $C$, as follows: (1) when $D=C-2 B$, (2) when $D=2 C-6 B$. In the first case we obtain the solution $y=\left(A+B x^{2}\right)^{1-c /(2 B)}$, and in the second case, $y=x\left(A+B x^{2}\right)^{1-c /(2 B)}$. 
Legendre's equation is obtained by making $A=1, B=-1, C=-2$, and $D=k(k+1)$.

\section{Professor W. M. Whyburn: Non-isolated critical points} of functions.

A function $f\left(x_{1}, \cdots, x_{n}\right)$ is studied, where the first partial derivatives of $f$ are assumed continuous in a closed and connected portion $R$ of the space of the $n$ real variables $x_{1}, \cdots, x_{n}$. Critical points of $f$ are considered under hypotheses which are not sufficient to cause these points to be isolated, and the notion of "critical sets" is developed. (For the case of isolated critical points, see Marston Morse, Transactions of this Society, vol. 27 (1925), pp. 345-396.) In particular, it is shown that if $f$ has two distinct "minimal sets" in $R$ it must have at least one other critical point in $R$. Upon specialization, this result yields Bieberbach's form of Birkhoff's "minimax principle" (see Bieberbach, Differentialgleichungen, Berlin, 1927, n. 140).

\section{Professor Philip Franklin: Equimomental systems.}

In this note we investigate certain simple configurations of mass points which are equimomental to any given rigid system, that is, have the same moment of inertia about all axes in space. It is shown that any system is equimomental to four mass points, which may be selected in a triple infinity of ways. The ratio of the masses at the points may be arbitrarily assigned, and in general uniquely determines the equivalent set. The tetrahedron with vertices at the four points has its opposite edges conjugate with respect to the homogeneous solid ellipsoid equimomental to the system. Some additional geometrical relations are given.

55. Mr. C. F. Muckenhoupt: Vibrating systems and almost periodic functions.

A vibrating system such as a vibrating string or membrane has its energy invariant under proper assumptions as to boundary conditions. By means of the finiteness of this invariant, together with similar in variants of higher order, it is proved that a vibrating membrane or string returns arbitrarily close to its initial position within some finite time. It follows from this with another definition of distance that the membrane or string not merely returns to the neighborhood of its initial position but that the difference between the displacement of the membrane or string and its original displacement has an energy which is arbitrarily small. From this the almost periodic character of the vibration may be proved. It is possible on this basis to discuss the closure of the set of characteristic functions of a vibration problem.

56. Professor Yusuke Hagihara: Complete stability of a dynamical system with long-period variations.

The author deals with a dynamical system with long-period variations superposed on short-period variations, from the stand-point of complete 
stability as defined by Birkhoff. Then a certain number of parameters are introduced in the differential equations of the dynamical system, and the results compared with Poincare's work in the problem of three bodies.

\section{Professor C. A. Garabedian: Concerning two-dimen-} sional solutions of the problem of the thick circular plate loaded at its center.

The author's corrections of de Saint-Venant's solutions (Journal de l'Ecole Polytechnique, (2), vol. 26 (1927)) followed the notation of de Saint-Venant and employed only devices found in the "Note du paragraphe 45." The present paper complements the foregoing in that it examines critically contributions subsequent to de Saint-Venant. Only by thus completing the study of the problem does it seem possible effectively to remove the obscurities which have pervaded the two-dimensional solutions. By including also some consideration of recently developed threedimensional solutions, there is secured, on this whole question of the thick circular plate centrally loaded, a perspective hitherto lacking.

58. Professor E. G. Keller: Harmonic functions for an anchor ring of elliptical meridian section. Preliminary communication.

In this paper a solution of Laplace's equation in series in elliptical toroidal coordinates is obtained. Circular symmetry is assumed. Each term of the series is a particular solution of the equation. Successive changes of independent variables and the same change of dependent variable as that employed by C. Neumann with reference to a circular tore (Theorie der Elektricitäts- und Wärme-Vertheilung in einem Ringe) yield the equation $\xi^{2} U_{\xi \xi}+2 \xi \eta U_{\xi \eta}+\eta^{2} U_{\eta \eta}+U_{\zeta \zeta}+\frac{1}{4} U=0$. A solution of this equation is $T_{n}(r) \cdot Y_{n}(\zeta)$, where $T_{n}$ and $Y_{n}$ are solutions of ordinary differential equations. The latter may be written as a series of Bessel coefficients of increasing order. The function $T_{1}$ is reducible to the Bessel function of order zero and of purely imaginary argument. Recurrence formulas for $T_{n}$ are obtainable. If an expansion of a function $f(r)$, in either of the series $\sum_{n=0}^{\infty} A_{n} T_{n}$ or $\sum_{=0}^{\infty} B_{m} T_{n}\left(a_{m} r\right)$, exists, the coefficients are readily expressed as definite integrals.

59. Dr. L. T. E. Thompson: Note on empirical summaries in engineering ballistics.

It is essential to facilitate extension (in scale) of experimental results bearing on certain ballistic problems. The study of wear or erosion phenomena, an aspect of the general uniformity problem of the gun cycle, may be developed by means of the ratio $f\left(C / C_{0}, l / d\right) \equiv P\left(C / C_{0}\right) / Z(l / d)$ $=E /\left(k d^{n}\right)$; rate of bore enlargement $E$, powder charge $C$ (datum value $C_{0}$ ), length and diameter of bore $l$ and $d ; k$ and $n$ are constants. Introducing appropriate assumptions with respect to maximum permissible enlargement, a relative life-of-gun formula is obtained. For the representation of plate punching data, it is practicable to identify a numerical (penetration) 
coefficient $F(e / d, \theta)=K_{1} M^{1 / 2} V \cos \theta / e^{1 / 2} d$; projectile mass $M$, diameter $d$, limit velocity $V$, plate thickness $e$, obliquity of impact $\theta$ (see the author's paper in Mémorial de l'Artillérie Française, vol. 6 (1927), p. 1255, note 3); by subsequent test this form is justified, especially in case application to moderate departure from similarity of projectiles is contemplated. It is required to determine one set of experimental $F$ curves for each class of projectiles and of plate characteristics. A laminated or divided armor coefficient can be identified by means of these and a corresponding absorption summary.

60. Professor Norbert Wiener: Hermitian polynomials and Fourier analysis.

The $n$th Hermitian function differs from its Fourier transform by the factor $i^{n}$. The present paper is devoted to the study of the transformation which multiplies the $n$th Hermitian function by $\omega^{n}$, where $\omega$ lies on the unit circle.

61. Mr. A. C. Berry: Spectral intensity of an n-dimensional function.

A proof of the complex $n$-dimensional form of the fundamental theorem in the Plancherel theory of the Fourier transform is given in the first part of the present paper. Although it has seemed necessary to use cartesian coordinates in giving the proof, the theorem itself is vectorial. The remainder of the paper discusses the intensity of the spectrum of a complex point function of a point in real $n$-dimensional space. A sufficient condition that the total spectral intensity be finite is given. This discussion is the vectorial, $n$-dimensional generalization of the first half of a recent article by N. Wiener (The spectrum of an arbitrary function, Proceedings of the London Mathematical Society, (2), vol. 27, No. 7).

\section{Mr. S. B. Littauer: On a theorem of M. Jacob.}

The author proves a theorem of Jacob concerning the equivalents of two forms of mean of a function by reducing this theorem to a particular case of a type of Tauberian theorem treated by Wiener. The reduction in one direction is immediate; in the other, a slight extension of Wiener's methods is necessary.

63. Professor O. D. Kellogg and Dr. F. Vasilesco: $A$ contribution to the study of a certain lemma in potential theory.

The lemma is as follows: every closed bounded set of points of positive capacity contains a regular point. It has been proved in the case of the logarithmic potential, but in space it is still in doubt. In order to make progress with the problem, it is necessary to have further knowledge of the properties of regular and exceptional points. In the present paper, the authors derive several new forms of criteria for regular and exceptional points. They show that the capacity of the portion of a set $E$ in a sphere of fixed radius about $P$ is a function of $P$ which is upper semicontinuous, 
and that a certain modified form of the series of Wiener is a function of $P$ which is of the second class, in the sense of Baire. In certain cases this function may reduce to one of the first class.

\section{Professor H. S. Vandiver: On Fermat's last theorem.}

In various papers published during the last fifteen years the writer has obtained a number of results concerning Fermat's last theorem. In the present paper all these results are obtained from two general methods of approaching the problem. Some new criteria are also derived by the use of these methods. When applied to special exponents they yield the result that if $n>2, x^{n}+y^{n}+z^{n}=0$ is impossible in rational integers $x, y$, and $z$, none zero, for every $n$ less than 211 .

\section{Professors H. S. Vandiver and Elizabeth T. Stafford:} Determination of some properly irregular cyclotomic fields.

The integer which expresses the number of classes of ideals in a cyclotomic field defined by $e^{2 \pi i / l, l}$ an odd prime, is generally written as the product of two factors which are also rational integers, called the first and the second factor of the class number respectively, that is, $h=h_{1} h_{2}$. A necessary but not a sufficient condition that $h_{2}$ be divisible by $l$ is that the first factor $h_{1}$ be divisible by $l$. In the present paper it is found by extensive computations that $h_{1}$ is divisible by $l$ for $l$ less than 211 only when $l=37$, $59,67,101,103,131,149,157$, and that $h_{2}$ is not divisible by $l$ in any of these cases. The paper includes proofs of the formulas employed, and descriptions of the many devices to shorten the computations.

66. Mr. H. T. Engstrom: Theorems on common index divisors of algebraic fields.

This paper gives the determination of common index divisors for algebraic fields of third, fourth, and fifth degrees. For these fields the common index divisor is defined by the prime ideal decomposition of the corresponding primes. For fields of ninth degree a special example is constructed showing that this is not the case in fields of ninth degree or higher.

\section{Professor W. L. G. Williams: On absolutely isobaric formal modular concomitants.}

The object of the present paper is to show that certain formal modular concomitants of binary forms are congruent to algebraic concomitants and to point out some applications of this fact in the theory of numbers. As a typical theorem the following may be cited: An absolutely isobaric formal modular invariant, $\bmod p$, a prime, of a binary form with binomial coefficients, no one of which is divisible by $p$, is congruent, $\bmod p$, to an algebraic invariant of this form. In this and other cases the author finds the algebraic concomitant to which a given formal modular concomitant is congruent. The following identical congruence will illustrate the applications to the theory of numbers. The coefficient of $x^{p-1} y^{p-1}$ in the expansion of $\left(a x^{2}+2 b x y+c y^{2}\right)^{k}(x \xi-y \eta)^{2 l}$ is congruent, $\bmod p$, to 
$K\left(a c-b^{2}\right)^{k-(p-1) / 2}\left(a \xi^{2}+2 b \xi \eta+c \eta^{2}\right)^{l}, \quad$ where $k, K$, and $l$ have the values $(p-1) / 2 \leqq k \leqq p-1, \quad K=(-1)^{(p-1) / 2} 2^{2 k}\left({ }_{l}^{2 l}\right)$, and $l=p-1-k$.

68. Professor Orrin Frink: The structure of finite algebras.

This paper deals with algebras with a finite number of elements, the addition group being abelian, but multiplication not necessarily commutative. Such algebras are important in the number theory of linear algebras. It is shown that such an algebra is reducible unless of prime power order. Most of the standard theorems of linear algebras go through, since their proofs do not depend on the existence of a scalar field, but on idempotents and the general theory of complexes. A special study is made of the important case of associative algebras of prime power order, which can be represented as algebras of matrices whose elements are residue classes of integers modulo a prime power.

\section{Professor Orrin Frink: $A$ theorem in boolean algebra.}

The theorem states that the result of substituting expressions which are in the completely expanded form in a function of several variables is given by multiplying the expressions together symbolically, preserving order, and interpreting any symbolic product which occurs as a coefficient in the result as meaning the result of substituting the factors of this product in the given function in the order in which they occur. It is shown how the theorem is used to simplify computation.

70. Dr. John Williamson: The complete system of two quadratic quaternary forms.

This paper obtains by a new method the system determined by $\mathrm{H}$. W. Turnbull (Proceedings of the London Mathematical Society, (2), vol. 18 (1919)). Starting from the complete system, already known (Turnbull and Williamson, Proceedings of the Royal Society of Edinburgh, vol. 45 (1925)), which involves three cogredient sets of point coordinates $x, y$, and $z$, it is shown how the variables $x, y$, and $z$ may be convolved to give a complete system of concomitants expressed in terms of point, line, and plane coordinates; that is, in terms of the variables $x, p=(x y)$, and $u=(x y z)$. While the results obtained are not new, the method has the advantage that it can be extended to finding, if not the complete system, at least a prepared system, for two quadratics in five or six variables. Incidentally, the number of irreducible concomitants obtained by Turnbull is diminished from 125 to 122 .

71. Dr. A. A. Albert (National Research Fellow): On the structure of normal division algebras.

It is first shown that in a division algebra if $y$ satisfies the minimum equation of $x$ then $y$ is a transform of $x$. The grade of an element is defined as the degree of its minimum equation. The type of an equation is defined as a function of the number of its ordinary complex roots rationally expressible in terms of one of them. The type of an element and that of an algebra are defined, and the structure of algebras of type $S_{k}$ is considered. 
The concept of "known algebras" is broadened and a necessary and sufficient condition that an algebra be a known algebra over a given subalgebra is found. It is demonstrated that if the direct product of two normal division algebras, each of the maximum type, is a division algebra, then it is a normal division algebra of the maximum type.

72. Dr. A. A. Albert: On the group of the rank function of a normal division algebra.

A new theorem in the Hilbert theory of irreducible polynomials whose coefficients are rational functions with coefficients in a field $K$ of several parameters is proved in this paper. The theorem states that, for any complex values of the parameters, the group of the resulting equation with respect to the field obtained by adjunction to $K$ of the resulting coefficients is a sub-group of the group of the original polynomial. This theorem is applied to the rank function of any normal division algebra, and it is shown that this function has the symmetric group. As a consequence of this it is seen that every normal division algebra in $n^{2}$ units contains an element whose minimum equation has degree $n$ and the symmetric group.

73. Dr. A. A. Albert: Normal division algebras in $4 p^{2}$ units, $p$ an odd prime.

Normal division algebras in $4 p^{2}$ units, $p$ an odd prime, are considered. Algebras of type $R_{3}$ are shown to be known algebras in the sense previously defined. A study is made of conditions that an algebra $A$ in $4 p^{2}$ units contain an element $x$ of grade $2 p$ whose minimum equation is $\phi\left(\omega^{2}\right)=0$. It is shown that $A$ is a known algebra over the $\Sigma$ algebra obtained from $x$ if and only if the equation $\varphi(\rho)=0$ is cyclic. This is the case considered by L. E. Dickson in his note in the September, 1928, number of this Bulletin. It is proved that these algebras are of type $R_{2 p}$ and hence not new.

\section{Dr. A. A. Albert: Normal division algebras in 36 units.}

In the present paper all normal division algebras in 36 units of type $R_{2}$ are shown by an elaborate argument to be of type $R_{3}$. The algebras of type $R_{3}$ were shown, in another paper, to be known algebras. A necessary and sufficient condition that the new algebras thus found be actually not equivalent to algebras previously constructed is given. Since the assumption of type $R_{2}$ is the weakest assumption of this kind that it is possible to make, the discussion is quite general.

\section{Professor Oystein Ore: An explicit case of "Zerle-} gungs"-groups.

Several of the general properties of the "Verzweigungs"-groups introduced by Hilbert are known, but except in the case of abelian fields, their structure is unknown. The author has shown in a previous paper that the prime ideals of a Galois field can be divided into two types, binomial and trinomial. For the binomial types the complete structure of the corresponding non-abelian Hilbert group is determined. 
76. Professor H. W. Brinkmann: On the group characteristics of multiply transitive permutation groups.

A permutation group on $n$ letters is, of course, a sub-group of the symmetric group on those letters. An irreducible homogeneous linear group isomorphic to the symmetric group thus furnishes a homogeneous linear group isomorphic to the given group. But this group is usually reducible. The present paper investigates the irreducible constituents of these groups. For example, it is shown that for a $t$-fold transitive group certain of these groups are irreducible. The irreducible ones at once give characteristics of the group in question.

77. Professor H. W. Brinkmann: Imaginary quadratic fields in which each genus contains but one class. Second paper.

In a previous paper (read at the summer meeting of the Society, 1928) fields containing two genera, each of which contains one class, were discussed. The case of fields with four such genera is taken up in the present paper. The conditions that a field be of this type are derived and applications are made to elementary number theory.

78. Professor H. W. Brinkmann: Note on algebraic equations not solvable by radicals.

It is well known that irreducible equations (with rational coefficients) exist for every degree which cannot be solved by radicals. It is the purpose of this note to give explicitly examples of such equations for prime degree. Thus we find that $x^{2}-p x+p=0$ is irreducible and cannot be solved by radicals for any prime $p \geqq 5$.

79. Professor Raymond Garver: On the removal of four terms from an equation by means of a Tschirnhaus transformation.

This paper has appeared in full in the January-February number of this Bulletin.

80. Professor Raymond Garver: Concerning certain functions of Brioschi.

A paper of Brioschi's (see his Opere, vol. 3, pp. 293-299) proves the existence, for an equation of odd degree $n$, of $(n-1) / 2$ polynomial functions possessing certain properties which make them of some value in connection with Tschirnhaus transformations. The present paper gives a simpler proof of the theorem, and considers its extension to equations of even degree.

81. Mr. R. E. Powers: Note on the Pellian equation.

In 1877 Artemas Martin found that the smallest integral value of $x$ which satisfies the Pellian equation $x^{2}-9817 y^{2}=1$ contains 97 digits (L. E. Dickson, History of the Theory of Numbers, vol. 2, p. 383). I have verified this result, and find that the corresponding value of $y$ contains 95 digits. 


\section{Professor D. C. Harkin: The abstract identity of modu-} lar systems and ideals.

By the method of abstraction and transformation by formal equivalence, Dedekind's theory of ideals (1871 and later) and Kronecker's theory of modular systems, as developed in his famous Grundzüge einer arithmetischen Theorie der algebraischen Grössen of 1882, are shown to be abstractly identical. A logically consistent theory of forms (or ideals) is built up, leading finally to the unique decomposition of forms into prime forms. The terminology and external form of the general theory developed agrees closely with Kronecker, but the root idea and fundamental meaning is taken almost bodily from Dedekind. Various possible interpretations of the abstract general theory are indicated : the theory of algebraic forms, with either the Gaussian transformation or the Kummer composition of forms as fundamental; geometrically, the correspondence of an ideal to a system of points on a fixed fundamental curve, a principal ideal to the complete intersection system of a fundamental curve with another algebraic curve, with equivalence translated into coresiduality.

\section{Professor R. L. Moore: On the structure of a continuum.}

It is shown that if $M$ is a bounded plane continuum and no upper semi-continuous collection of mutually exclusive subcontinua of $M$ is homeomorphic with a domain, then there exists an upper semi-continuous collection $G$ of mutually exclusive continua filling up $M$ such that $G$ is a dendron with respect to its elements and such that if $H$ is another collection satisfying these conditions then every element of $H$ is made up of elements of $G$. The elements of $G$ will be called dendratomic subsets of $M$. For each non cut point $P$ of the bounded plane continuum $M$ let $M_{P}$ denote the set of all points $X$ of $M$ such that $X$ is not separated from $P$ in $M$ by any one point. For each cut point $P$ of $M$ let $M_{P}$ denote $P$. If $M$ is a continuous curve every $M_{P}$ is a cyclic element of $M$ in the sense of G. T. Whyburn and if $Q$ is an upper semi-continuous collection of mutually exclusive continua filling up $M$ and no $M_{P}$ contains points of two different continua of $Q$ then every continuum of $Q$ is made up of continua of the collection $G_{1}$ defined on page 700 of volume 34 of this Bulletin.

84. Professor R. G. Lubben: The metrization problem and complete spaces.

The author shows that certain well known conditions for the metrization of a topological space may be strengthened so as to give necessary and sufficient conditions that such a space be complete. It follows from this result that a locally compact, metric space is complete. A universal complete enclosure of a metric space $M$ is by definition a complete space $N$ which contains $M$ and admits a metric $d$ such that if $T$ is any complete space whatever containing $M$, and $d^{\prime}$ is a metric with reference to which $T$ is complete, then if a sequence of points in $M$ is a fundamental sequence with reference to $d^{\prime}$ it is a fundamental sequence with reference to $d$. In order that a metric space admit a universal complete enclosure, it is necessary 
and sufficient that it be separable; in order that it admit a minimal universal complete enclosure, it is necessary and sufficient that it be locally compact and separable.

85. Professor R. G. Lubben: Concerning the distributive property.

The author establishes the following results. (1) If a space $L$ has the distributive property (see Transactions of this Society, vol. 30 (1928), p. 669), then every point set in it having the power of the continuum has a limit point. (2) In a space $S$ having the distributive property, every point set having the power of the continuum contains a limit point of itself. (3) If a space $S$ has the distributive property, then every regular, locally compact sub-space of it has this property. (4) Every space $S$ which has the distributive property and satisfies the first countability axiom is regular and locally compact. (5) A space $S$ which is regular, is locally compact, and has the Lindelöf property, has the distributive property.

86. Mr. J. H. Roberts: On a problem of C. Kuratowski's concerning upper semi-continuous collections.

In a letter to R. L. Moore dated April 9, 1927, C. Kuratowski raised the question as to whether or not there exists an upper semi-continuous collection $X$ of mutually exclusive continua no one of which is a point such that (1) the sum of the continua of the collection $X$ fills a square plus its interior, and (2) if each continuum of the collection $X$ is regarded as a point, the space so obtained is in continuous one-to-one correspondence with a square plus its interior. In the present paper this question is answered in the affirmative.

87. Mr. J. H. Roberts: Upper semi-continuous collections of continua some of which are unbounded.

R. L. Moore has proved that if, in a plane $S, G$ is an upper semi-continuous collection of mutually exclusive bounded continua no one of which separates $S$ and such that every point of $S$ belongs to some continuum of $G$, then, if the continua of $G$ are regarded as points, the space so obtained is in continuous one-to-one correspondence with the plane $S$. In the present paper it is shown that if $G$ is an upper semi-continuous collection of mutually exclusive continua such that (1) every point of the plane $S$ belongs to some continuum of $G,(2)$ at least two continua of $G$ are unbounded, and (3) the space of elements of $G$ is metric, then the space of elements of $G$ is in continuous one-to-one correspondence with a subset of the plane $S$.

88. Dr. W. L. Ayres (National Research Fellow): Continuous curves in which every arc may be extended.

An $\operatorname{arc} \alpha$ of a set $K$ is said to be extendible in both directions in $K$ if $K$ contains an arc $\beta$ such that every point of $\alpha$ is an interior point of $\beta$. It is shown in this paper that a plane continuous curve $M$ has the property that every arc is extendible in both directions in $M$ if and only if one of the two following conditions holds: (a) no points of $M$ may be $\epsilon$-separated by an acyclic continuous curve; (b) the sum $B$ of the boundaries of the 
complementary domains of $M$ is closed and every point of $B$ is either a cut point of $M$ or belongs to the boundaries of at least two complementary domains of $M$. If either (a) or (b) is true, then every point of $M$ is a point of $B$. For any point $P$ of $M$ and any positive number $\epsilon$, there exists a simple closed curve $J$ of diameter less than $\epsilon$ and enclosing $P$ and such that the set $M \cdot J$ consists of a finite number of components.

89. Dr. W. L. Ayres: On generalizations of continuous curves.

If $x_{i}(i=1,2, \cdots, n)$, is a continuous function of $t$, the set of all points $\left(x_{1}, x_{2}, \cdots, x_{n}\right)$ for $0 \leqq t \leqq 1$ is called a continuous curve. Two possible generalizations of this notion may be had by allowing $t$ to take on all values or all values $t \geqq 0$. In this note it is shown that these two generalizations are equivalent and the type is characterized.

90. Professor G. T. Whyburn: Cut points of connected sets and of continua.

Let $M$ be any continuum (in a locally compact, metric, and separable space), let $A$ and $B$ be any two mutually exclusive closed subsets of $M$, let $K$ be the set of all those points of $M$ which separate $A$ and $B$ in $M$, and let $G$ denote the set of all cut points of $M$. In this paper it is shown that (1) $K+A+B$ contains every point of $\bar{K}$ which is a Menger regular point of $M+P$; (2) $K$ is the sum of a $G_{\delta}$ set and a countable set; (3) $G$ is a $G_{\delta \sigma}$, that is, the sum of a countable number of $G_{\delta}$ sets; (4) if $K$ is uncountable, it contains a perfect set; (6) if $M$ is compact and irreducible between $A$ and $B$, then $K$ contains every point of $M-(A+B)$ at which $M$ is connected im kleinen. Result (1) is true for any connected set $M$. Some other theorems of a similar character are also obtained.

91. Professor G. T. Whyburn: On the structure of connected and connected im kleinen point sets.

It is shown in this paper that all connected and connected im kleinen point sets $M$ in a locally compact, metric, and separable space admit of decomposition into elements highly analogous to the cyclic elements of a continuous curve. Elements $(a)$ of such a set $M$ are connected subsets of $M$ which have no cut point and which are saturated with respect to this property; elements (b) of $M$ are cut points of $M$; and elements $(c)$ of $M$, called end points, are points $P$ of $M$ such that a sequence $V$ of points of $M$ exists converging to $P$ and such that each point of $M$ distinct from $P$ is separated in $M$ from $P$ by all save a finite number of points of $V$. These correspond respectively to the maximal cyclic curves, cut points, and end points of a continuous curve; and it is shown that the structure of any such set $M$ with respect to its elements $(a),(b)$, and $(c)$ is very similar to the structure of a continuous curve with respect to its cyclic elements (see American Journal of Mathematics, vol. 50, pp. 167-194). One interesting theorem proved is that every connected subset of the set of all elements of classes $(b)$ and $(c)$ is arcwise connected. 


\section{Professor G. T. Whyburn: Continuous curves and arc sums.}

Menger has suggested the problem of characterizing a continuous curve which is the sum of a countable number of simple continuous arcs. In this connection the following theorems are proved. (1) The continuous curve $M$ is the sum of a countable number of arcs if and only if (a) the end points of $M$ are countable, and (b) each maximal cyclic curve of $M$ is the sum of a countable number of arcs. (2) The boundary $N$ of a complementary domain of a plane continuous curve is the sum of a countable number of arcs if and only if the end points of $N$ are countable. (3) The set $K$ of all the local cut points of any continuous curve $M$ is a subset of the sum of a countable number of arcs which lie in $M$; and if $M-K$ is countable, $M$ is the sum of a countable number of arcs.

\section{Professor James Pierpont: On the motion of a rigid body about a fixed point in space of constant curvature.}

The first to consider the motion of a solid in elliptic space was Clifford, in a paper read before the London Mathematical Society in 1874, but not published until 1882 . This was followed by a paper by R. S. Heath published in 1884 in the Transactions of the Royal Society of London. Finally, de Francesco treated this problem in two papers in the Atti of the Academy of Science of Naples in 1900. Clifford employs biquaternions, Heath and de Francesco use the six homogeneous coordinates of a right line tied down by two biquadratic relations; de Francesco considers hyperbolic as well as elliptic space, and he also considers in detail special cases, which Heath does not do. The present author treats another special case, viz., the Maxwell top in space of constant curvature. In this important case he shows that homogeneous line coordinates are quite unnecessary, from which follows a great gain on the analytical side of the problem. With the same degree of accuracy as employed in classical mechanics we have the notion of center of mass. From this it results that the motion of the top in elliptic (hyperbolic) space would appear to an observer in euclidean space as that of a top with certain assigned moments of inertia and a certain center of mass.

94. Dr. A. J. Lotka: Notes on Volterra's mathematical theory of biological associations.

Volterra has set up a system of differential equations to represent the course of events in a system comprising two biological species competing for a common food supply. He has discussed certain properties of the functions defined by these equations, but has not offered any general solution. Such a solution in form of an exponential series is now given, the case discussed by Volterra being a special case of the general theory of the growth of $n$ populations living together in a common environment, as developed in prior publications by the present author. The case discussed by Volterra may also be regarded as the Verhulst-Pearl law of growth for two competing populations. The independent variable $t$ (time) can be 
eliminated from the system of differential equations, which then contains only two variables. The isoclines of the integral curves of the system are investigated, and a map of the family of integral curves is prepared by their aid. The discussion of some other cases treated by Volterra is reserved for a future occasion. Application of the method of analysis to economic problems (for example, competition of two or more producers supplying the same market) is suggested.

95. Dr. P. R. Rider: On the moments of the frequency distribution of third moments.

This paper develops formulas for the moments of the frequency distribution of third moments of samples of $n$ from an infinite population. The first and second moments are given for any type of population, the third and fourth moments for a normal population.

96. Dr. P. R. Rider: The effect of skewness in the sampled population on the frequency distribution of the ratio of mean to standard deviation in small samples. Preliminary communication.

The frequency distribution of the ratio of mean to standard deviation in samples of $n$ from a normal population has been rather thoroughly discussed by "Student" and others. In samples from a normal population the mean and the square of the standard deviation are uncorrelated; for other types of population correlation between them exists. Shewhart and Winters have made an experimental study of the distribution of the ratio of mean to standard deviation in samples from certain non-normal populations, and have given their results in a recent number of the Journal of the American Statistical Association. The present paper endeavors to shed more light on the effect of skewness in the sampled population on the distribution of this ratio.

97. Professor A. H. Copeland: A proof that almost every number is admissible and is associated with the probability one half.

In this paper it is proved that in a certain sense almost every event satisfies the fundamental assumptions of the theory of probability, and moreover almost every event has the probability one-half. The sense in which this statement is true is as follows. If an event behaves in accordance with the assumptions of the theory of probability, then the history of successes and failures of this event must be represented by an admissible number. The admissible numbers all lie in the interval from zero to one, and the measure of the set of admissible numbers is equal to one. That is, almost every number in the unit interval is admissible. Furthermore it is proved that almost every admissible number is associated with the probability one-half. 
98. Professor C. F. Roos: A mathematical theory of the business cycle.

Many economists have empirically investigated the changes in prices over a period of time, and have evolved an empirical theory generally known as the theory of the business cycle, which, in brief, states that prices are periodic functions of the time. Other equally competent investigators have branded this theory as a myth, and have exhibited empirical curves to support their contentions. The present paper gives a purely theoretical discussion of prices, and shows that these views, rather than being contradictory, are both only partial manifestations of an underlying theory. The hypothesis that a producer endeavors to determine his price so that he obtains a maximum profit over a period of time requires that the price satisfy a certain differential equation. This differential equation has ten distinct types of solutions, all of which seem to have economic significance. A truly periodic solution supporting the theory of the business cycle can result only if interest is neglected. Conditions are obtained that will lead to the ten different types of solutions; all the results are stated in a form that will allow experimental investigation.

99. Mr. H. H. Pixley: A theory of discontinuous price and production curves.

In this paper the author considers a generalization of Roos's problem of depreciation and replacement in the mathematical theory of economics, and removes the restrictions of continuity of price-time and rate of productiontime curves at the time of replacement. Some applications are made of the general mathematical theory given in the author's earlier paper Discontinuous solutions in the problem of depreciation and replacement, in considering special problems believed to have actual economic significance. The present paper consists essentially of applications.

R. G. D. RichaRdSON, Secretary 